for

\title{
Orbital-Overlap Control in the Solid-State Reactivity of $\beta$-Azido-Propiophenones: Selective Formation of cis-Azo-Dimers
}

Jagadis Sankaranarayanan, Lauren Bort, Sarah M. Mandel, Ping Chen, Jeanette A. Krause, Elwood E. Brooks, Pearl Tsang, Anna D. Gudmundsdottir*

Department of Chemistry, University of Cincinnati, Cincinnati, Ohio 45221-0172

Anna.Gudmundsdottir@uc.edu

\section{Table of Contents}

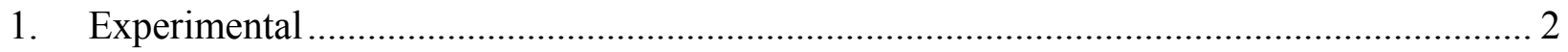

1.1 Preparation of Starting Materials. ......................................................................... 2

1.2 Solid State Photolysis of Azides 1. ........................................................................ 2

1.3 Raman Spectroscopy: ....................................................................................... 2

1.4 Solid-State ${ }^{13}$ C NMR Spectroscopy ............................................................... 5

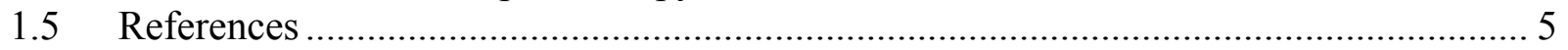

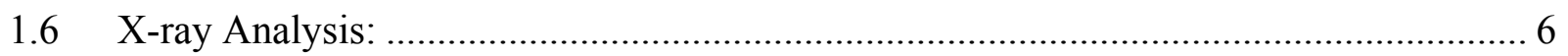

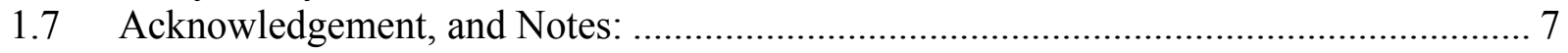

1.8 Table 1. Crystal data and structure refinement ..................................................... 7

1.9 Table 2 Bond lengths $[\AA]$, bond angles $\left[{ }^{\circ}\right]$ and torsion angles $\left[{ }^{\circ}\right]$............................. 8

1.10 Table 3. N...N Intermolecular Interactions $(\AA)$................................................ 9

1.11 Table 4. Least Squares Planes ...................................................................... 10

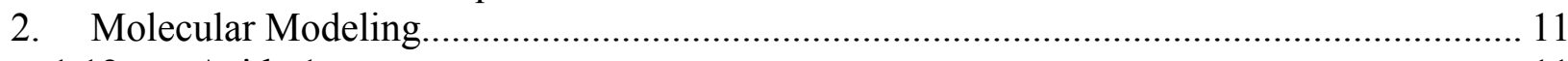

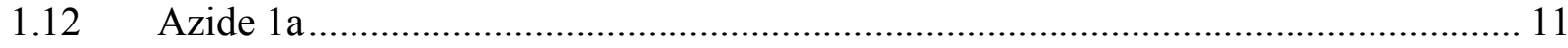

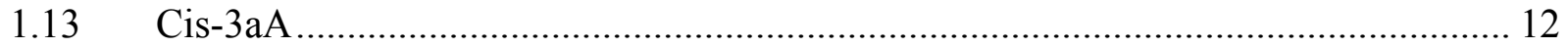

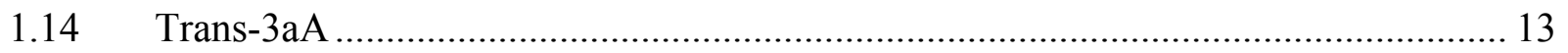

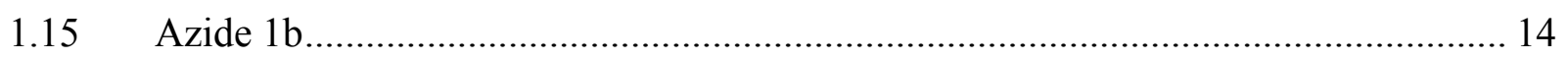

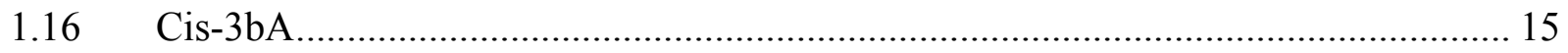

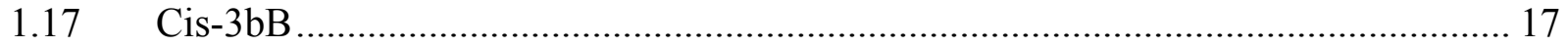

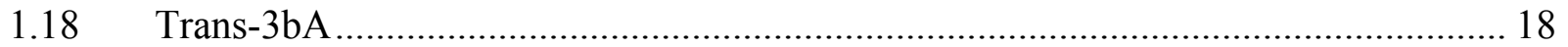

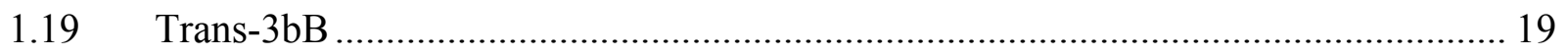

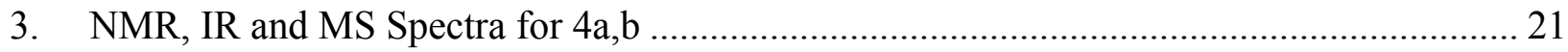




\section{Experimental}

\subsection{Preparation of Starting Materials.}

Azides 1a,b were prepared as we have described previously. ${ }^{1}$

\subsection{Solid State Photolysis of Azides 1.}

A procedure analogous to that adopted by Garcia-Garibay and co-workers was followed. ${ }^{3}$ In a typical procedure, crystals of azide $1 \mathrm{~b}(600 \mathrm{mg}, 2.3 \mathrm{mmol})$ were taken in $500 \mathrm{~mL}$ of de-ionized water and purged with argon for 20 mins. This suspension was stirred while externally cooled to $1^{\circ} \mathrm{C}$ and photolyzed for $24 \mathrm{hrs}$ during which the white crystals of the azide acquired an orangeyellow color. Samples were withdrawn periodically and the reaction was monitored using IR spectroscopy. After photolysis the suspension was filtered and water was removed from the sample by lyophilization. HPLC analysis of the irradiated crystals dissolved in ethyl acetate, showed formation of $\mathbf{4 b}$ and remaining starting material in the ratio of $86: 14$, respectively.

The spectroscopy characterization of $\mathbf{4 b}$ are as follows: 1-(4-bromophenyl)-3-[5-(4-bromophenyl)pyrazol-1-yl]-propan-1-one, (50 mg, $0.115 \mathrm{mmol}, 10 \%$ yield).

IR $\left(\mathrm{CHCl}_{3}\right) 2922,2848,1682,1582,1452,1395 \mathrm{~cm}^{-1}$

${ }^{1} \mathrm{H}$ NMR $\left(250 \mathrm{MHz}, \mathrm{CDCl}_{3}\right): 3.58(\mathrm{t}, 2 \mathrm{H}, 7 \mathrm{~Hz}), 4.51,(\mathrm{t}, 2 \mathrm{H}, 7 \mathrm{~Hz}), 6.27,(\mathrm{~s}, 1 \mathrm{H}), 7.33(\mathrm{~d}, 2 \mathrm{H}$, $8 \mathrm{~Hz}), 7.5-7.6(\mathrm{~m}, 4 \mathrm{H}), 7.78(\mathrm{~d}, 2 \mathrm{H}, 8 \mathrm{~Hz}) \mathrm{ppm} .{ }^{13} \mathrm{C}-\mathrm{NMR}\left(100 \mathrm{MHz}, \mathrm{CDCl}_{3}\right): \delta 196.4,142.8$, 139.2 , 135.0, 132.0, 131.9, 130.5, 129.6, 129.5, 128.7, 123.0, 106.5, 44.3, 38.3 ppm. MS (ESI $\mathrm{M}+\mathrm{H})$ : Calculated for $\mathrm{C}_{18} \mathrm{H}_{14} \mathrm{~N}_{2} \mathrm{OBr}_{2}$. Calculated: 434.9551 ; Found 434.9498.

We have previously published the spectroscopic characterization of $\mathbf{4 a}{ }^{3}$

\subsection{Raman Spectroscopy:}

Raman experiments were performed in collaboration with Dr.Punit Boolchand at the Department of Electrical Engineering University of Cincinnati. Raman scattering experiments were performed on solid samples using a Thermo-Nicolet NEXUS 870 FTIR research bench with an FT-Raman Module. Typically, samples were mounted on a view stage in macro sampling mode and about $650 \mathrm{~mW}$ of laser power (1064 $\mathrm{nm}$ radiation from a $\mathrm{Nd}$ :YAG laser) with a $400 \mu \mathrm{m}$ spot size was used to excite the scattering. An InGaAs detector was used to accumulate a spectrum with a typical run making use of 1000 scans $\left(4 \mathrm{~cm}^{-1}\right.$ resolution $)$.

The sample after photolysis has a low melting point $\left(40-60^{\circ} \mathrm{C}\right)$, and so $260 \mathrm{~mW}$ of laser power was used while the sample was cooled to $78 \mathrm{~K}$ in a Joule-Thompson refrigerator. For this sample an accumulation of 2000 scans was needed 


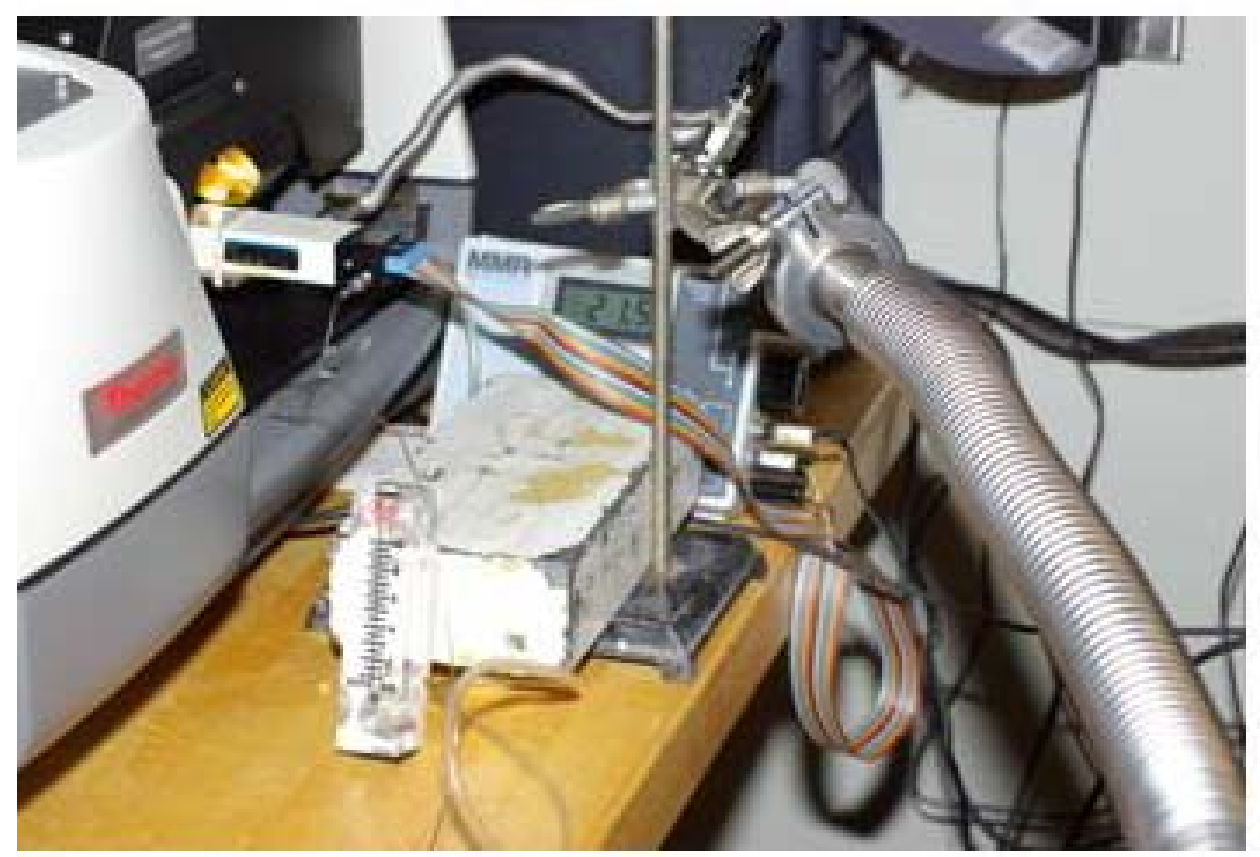

A picture of the Raman View Stage and Joule Thompson refrigerator (white box) being pumped by a vacuum line. 

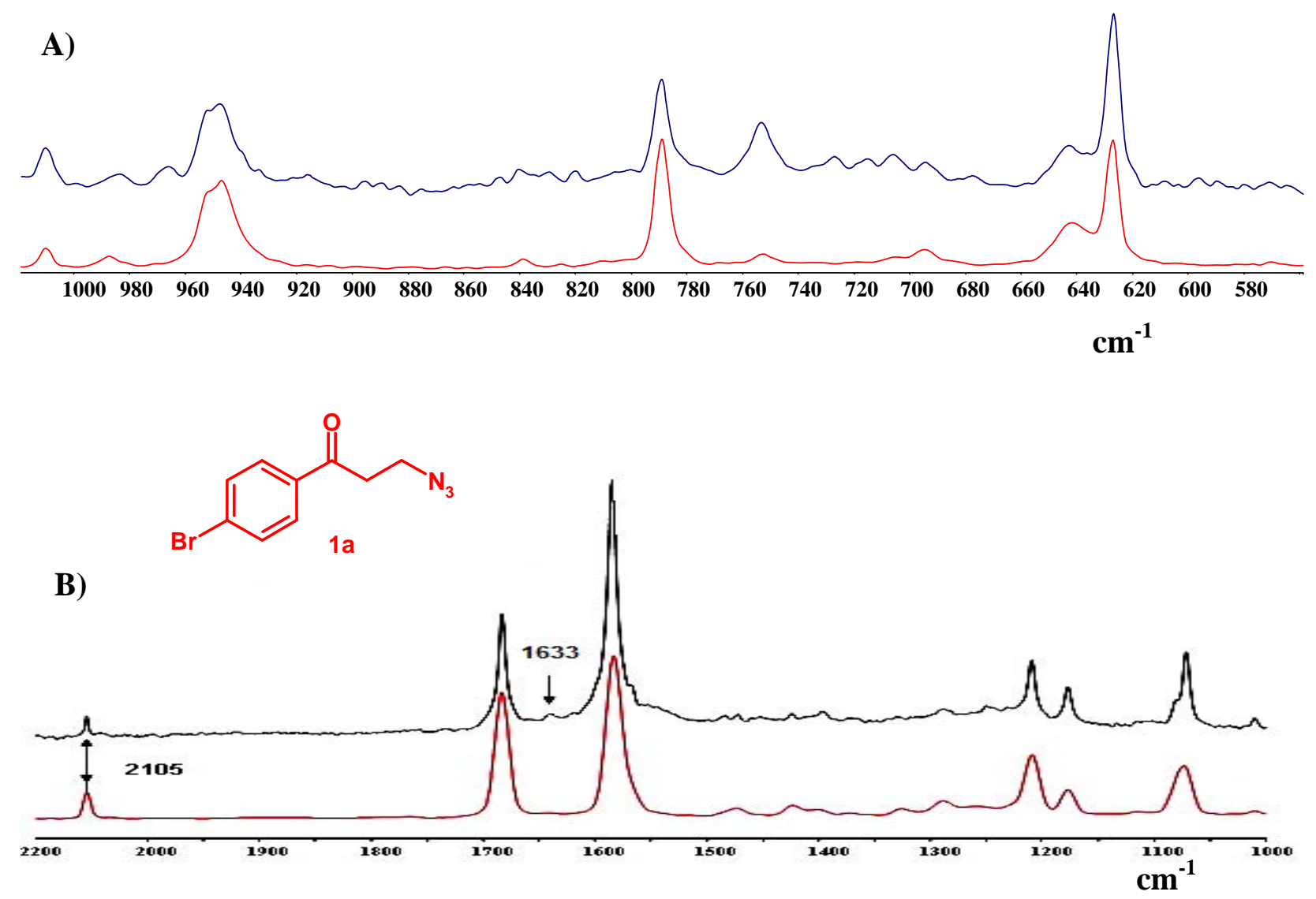

Figure S1. Expansion of raman spectra of azide $\mathbf{1 b}$ before (red) and after irradiation A) blue and B) black.

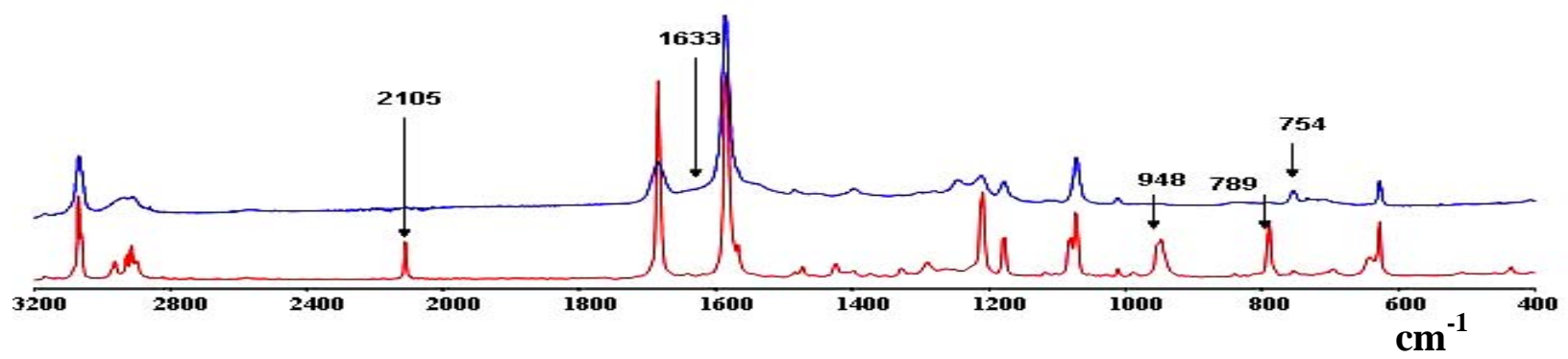

Figure S2. Raman Spectra of azide 1b before (red) and after irradiation (blue) at higher conversion than in Figure S1. 

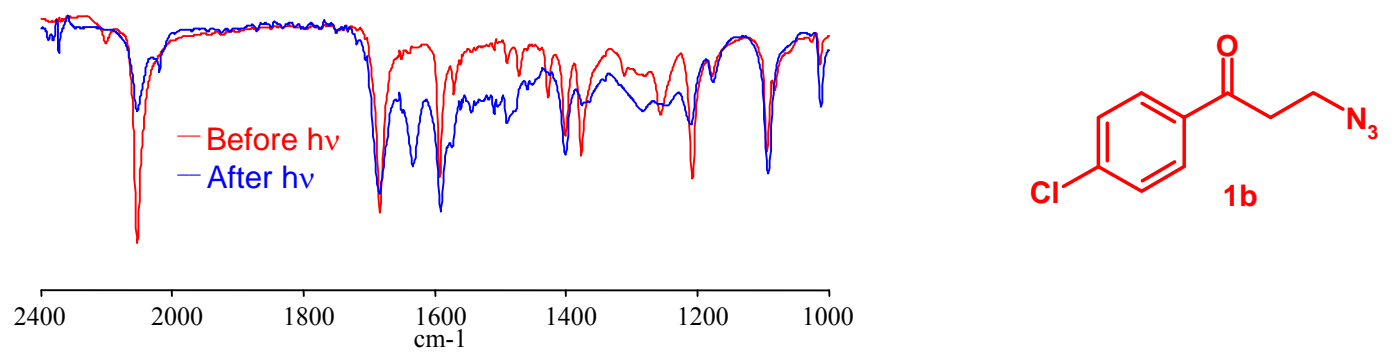

Figure S3. IR spectra of azide before (red) and after irradiation (blue).

\subsection{Solid-State ${ }^{13}$ C NMR Spectroscopy}

The solid state NMR experiments were acquired on a Bruker AMX $400 \mathrm{MHz}$ wide bore Spectrometer and high frequency broadband probe. The CPMAS-TOSS (Total Sideband Supression) Bruker pulse sequence and a $5 \mathrm{kHz}$ sample spin speed were employed. ${ }^{4}$

The data were acquired using 2048 total points. The ${ }^{13} \mathrm{C} 180$ degree pulse length was 7.5 usec while the 90 degree ${ }^{1} \mathrm{H}$ pulse length was 6.5 usec. All spectra were recorded using 14,332 acquisitions with a recycle delay of 4.0seconds; each spectrum required approximately 16 hours to acquire. The ${ }^{13} \mathrm{C}$ chemical shift values were referenced relative to the alpha carbon resonance of glycine (43.3 ppm).

\subsection{References}

1. Singh, P. N. D.; Muthukrishnan, S.; Murthy, R. S.; Klima, R. F.; Mandel, S. M.; Hawk, M.; Yarbrough, N.; Gudmundsdottir, A. D. Tetrahedron Lett. 2003, 51, 9169.

2. Sankaranarayanan, J.; Mandel, S. M.; Krause, J. A.; Gudmundsdottir, A. D. Acta Crystallogr., Sect. E, 2007, E63, o721.

3. Veerman M., Resendiz M. J. E. ;Garcia-Garibay, M.A. Org. Lett., 2006, 8, 2615 -2617.

4. Dixon, W. T.; Schaefer, Jacob; Sefcik, M. D.; Stejskal, E. O.; McKay, R. A. J. Mag, Res. 1982, 49(2), 341-5 


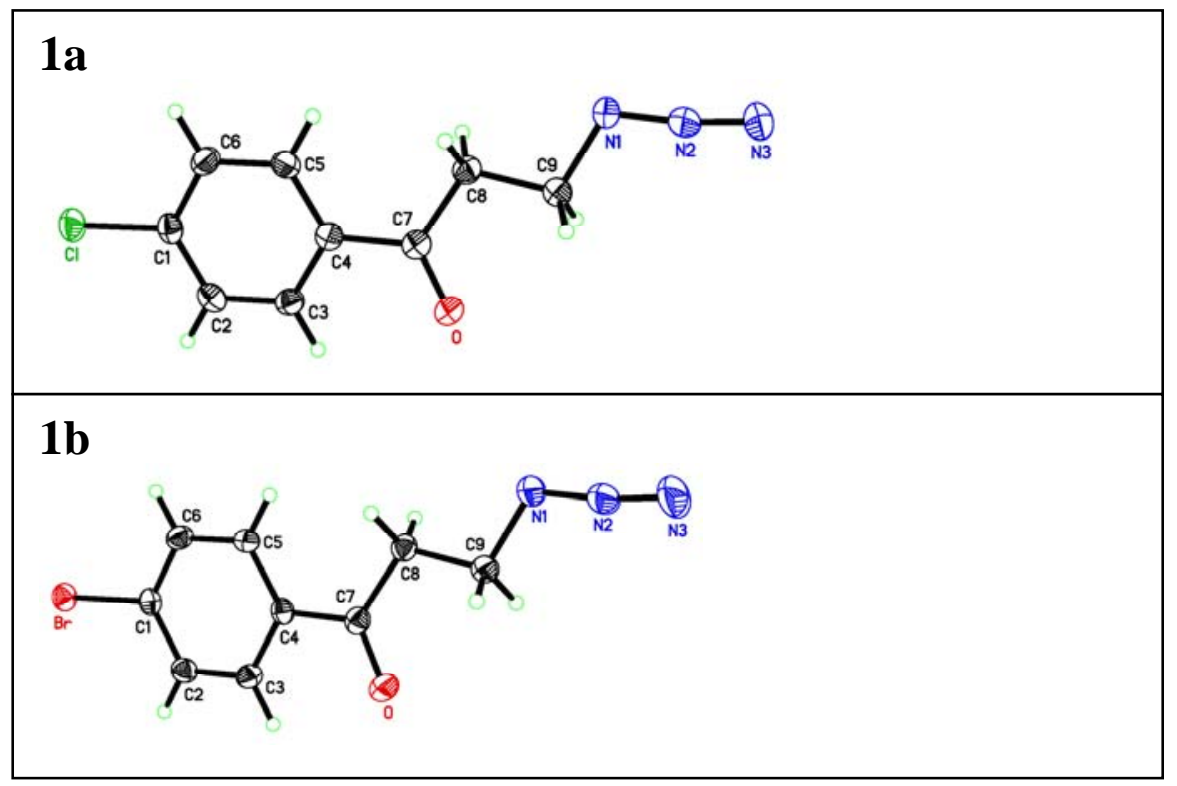

Figure S4. X-ray Structure of Azides 1a,b.

\subsection{X-ray Analysis:}

Single crystals of $\mathbf{1 a}$ and $\mathbf{1 b}$ were obtained from EtOAc-hexane and $\mathrm{CHCl}_{3} /$ hexane, respectively. For X-ray examination and data collection, suitable crystals were mounted on the tip of a glass fiber or a Cryo-loop with paratone-N oil and transferred immediately to the goniometer bathed in a cold stream.

Intensity data for 1a were collected on a SMART6000 CCD diffractometer while data for 1b were collected on a SMART $1 \mathrm{~K}$ CCD diffractometer. In both cases, graphite-monochromated Mo $\mathrm{K} \alpha \forall$ radiation $(\lambda=0.71073 \AA)$ was employed and data was collected at $150 \mathrm{~K}$. For data collection, frames were measured at $0.3^{\circ}$ intervals of $\omega$ and out to $\sim 28^{\circ}$ in $\theta$. The data frames were processed using the program SAINT. The data were corrected for decay, Lorentz and polarization effects. An absorption correction based on the multi-scan technique and beam corrections were applied using SADABS.

The structure of $\mathbf{1 b}$ was solved by direct methods while the Patterson method was used for 1a. Both structures were completed with the difference Fourier technique and refined by fullmatrix least squares on $\mathrm{F}^{2}$. Non-hydrogen atoms were refined with anisotropic displacement parameters. All $\mathrm{H}$-atom positions were calculated; a riding model was used in subsequent refinements. The isotropic temperature factors for the $\mathrm{H}$-atoms were defined as $\mathrm{Uiso}=1.2 \mathrm{U}(\mathrm{C})$. The refinement converged with crystallographic agreement factors as summarized in Table 1. 


\subsection{Acknowledgement, and Notes:}

(1) Funding for the SMART6000 diffractometer was through NSF-MRI grant CHE-0215950. Data collection on the SMART1K diffractometer was through the Ohio Crystallographic Consortium, funded by the Ohio Board of Regents 1995 Investment Fund (CAP-075) located at the University of Toledo, Instrumentation Center in A\&S, Toledo, OH 43606.

(2) SMART v5.054, v5.628 and SAINT v5.A06, v6.28A programs were used for data collection and data processing, respectively. Bruker Analytical X-ray Solutions, Inc., Madison, WI. SADABS v2.03 was used for the application of semi-empirical absorption and beam corrections. G. M. Sheldrick, University of Göttingen, Germany. SHELXTL v5.03, 6.12, v6.14 were used for the structure solution and generation of figures and tables. G. M. Sheldrick, University of Göttingen, Germany and Bruker Analytical X-ray Solutions, Inc., Madison, WI. Neutral-atom scattering factors were used as stored in this package.

\subsection{Table 1. Crystal data and structure refinement}

\begin{tabular}{lll} 
& \multicolumn{1}{c}{$\mathbf{1 a}$} & \multicolumn{1}{c}{$\mathbf{1 b}$} \\
Empirical formula & $\mathrm{C}_{9} \mathrm{H}_{8} \mathrm{~N}_{3} \mathrm{OCl}$ & $\mathrm{C}_{9} \mathrm{H}_{8} \mathrm{~N}_{3} \mathrm{OBr}$ \\
Formula weight & 209.63 & 254.09 \\
Temperature, $\mathrm{K}$ & $150(2)$ & $150(2)$ \\
Wavelength, $\AA$ & 0.71073 & 0.71073 \\
Crystal system & Triclinic & Triclinic \\
Space group & $\mathrm{P}-1$ & $\mathrm{P}-1$ \\
$\mathrm{a}, \AA$ & $3.8979(3)$ & $3.9521(3)$ \\
$\mathrm{b}, \AA$ & $8.6911(7)$ & $8.8020(6)$ \\
$\mathrm{c}, \AA$ & $13.8933(11)$ & $13.9895(9)$ \\
$\alpha{ }^{\circ}$ & $94.795(2)$ & $94.508(2)$ \\
$\beta,^{\circ}$ & $94.495(2)$ & $95.291(2)$ \\
$\gamma,^{\circ}$ & $95.972(2)$ & $95.818(2)$ \\
Volume $^{\circ}\left(\AA^{3}\right), \mathrm{Z}$ & $464.67(6), 2$ & $480.17(6), 2$ \\
Density (calculated), $\mathrm{Mg} / \mathrm{m}^{3}$ & 1.498 & 1.757 \\
Absorption coefficient, $\mathrm{mm}^{-1}$ & 0.378 & 4.248 \\
$\mathrm{~F}(000)$ & 216 & 252 \\
Crystal size, mm & $0.45 \times 0.35 \times 0.15$ & $0.38 \times 0.20 \times 0.02$ \\
$\theta$ range for data collection, ${ }^{\circ}$ & 1.48 to 23.26 & 2.65 to 28.27 \\
Limiting indices & $-4<\mathrm{h}<4$ & $-5<\mathrm{h}<5$ \\
& $-9<\mathrm{k}<9$ & $-11<\mathrm{k}<11$ \\
& $-15<1<15$ & $-18<1<18$ \\
Reflections collected & 4158 & 5050 \\
Independent reflections & $1329\left(\mathrm{R}_{\text {int }}=0.0196\right)$ & $2316\left(\mathrm{R}_{\text {int }}=0.0311\right)$ \\
Completeness to $\theta=28.27^{\circ}$ & $98.8 \%$ & $96.9 \%$ \\
Absorption correction & Multi-scan & Multi-scan \\
Max. and min. transmission & 0.9544 and 0.8484 & 0.9198 and 0.2951
\end{tabular}


Refinement method

Data / restraints / parameters

Goodness-of-fit on $\mathrm{F}^{2}$

Final $R$ indices $[\mathrm{I}>2 \sigma(\mathrm{I})]$

$\mathrm{R}$ indices (all data)

Largest diff. peak and hole, $\mathrm{e} \AA^{-3}$
Full-matrix least-squares on $\mathrm{F}^{2}$

$\begin{array}{ll}1329 / 0 / 127 & 2316 / 0 / 127 \\ 1.063 & 0.981 \\ \mathrm{R} 1=0.0289 & \mathrm{R} 1=0.0338 \\ \mathrm{wR} 2=0.0755 & \mathrm{wR} 2=0.0751 \\ \mathrm{R} 1=0.0314 & \mathrm{R} 1=0.0454 \\ \mathrm{wR} 2=0.0782 & \mathrm{wR} 2=0.0782 \\ 0.487 \text { and }-0.224 & 0.582 \text { and }-0.682\end{array}$

\subsection{Table 2 Bond lengths $[\AA]$, bond angles $\left[{ }^{\circ}\right]$ and torsion angles $\left[{ }^{\circ}\right]$}

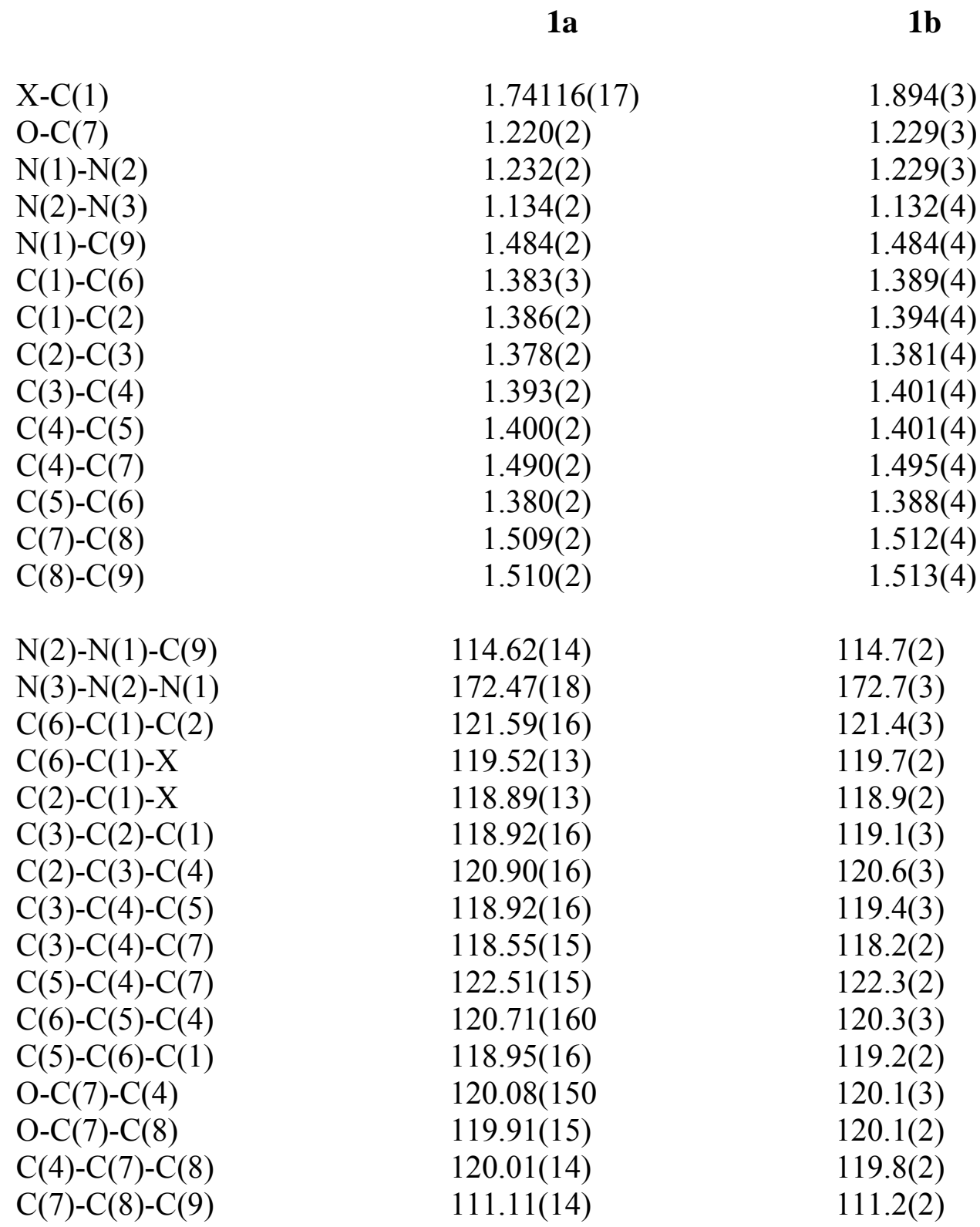




\begin{tabular}{lcc}
$\mathrm{N}(1)-\mathrm{C}(9)-\mathrm{C}(8)$ & $108.22(14)$ & $108.1(2)$ \\
$\mathrm{C}(9)-\mathrm{N}(1)-\mathrm{N}(2)-\mathrm{N}(3)$ & $-179.7(15)$ & $180(3)$ \\
$\mathrm{C}(6)-\mathrm{C}(1)-\mathrm{C}(2)-\mathrm{C}(3)$ & $1.3(3)$ & $1.6(4)$ \\
$\mathrm{X}-\mathrm{C}(1)-\mathrm{C}(2)-\mathrm{C}(3)$ & $-177.87(13)$ & $-177.40(19)$ \\
$\mathrm{C}(1)-\mathrm{C}(2)-\mathrm{C}(3)-\mathrm{C}(4)$ & $-1.1(3)$ & $-1.3(4)$ \\
$\mathrm{C}(2)-\mathrm{C}(3)-\mathrm{C}(4)-\mathrm{C}(5)$ & $0.0(3)$ & $0.0(4)$ \\
$\mathrm{C}(2)-\mathrm{C}(3)-\mathrm{C}(4)-\mathrm{C}(7)$ & $178.23(14)$ & $178.2(2)$ \\
$\mathrm{C}(3)-\mathrm{C}(4)-\mathrm{C}(5)-\mathrm{C}(6)$ & $0.9(3)$ & $1.0(4)$ \\
$\mathrm{C}(7)-\mathrm{C}(4)-\mathrm{C}(5)-\mathrm{C}(6)$ & $-177.27(15)$ & $-177.1(2)$ \\
$\mathrm{C}(4)-\mathrm{C}(5)-\mathrm{C}(6)-\mathrm{C}(1)$ & $-0.7(3)$ & $-0.7(4)$ \\
$\mathrm{C}(2)-\mathrm{C}(1)-\mathrm{C}(6)-\mathrm{C}(5)$ & $-0.4(3)$ & $-0.6(4)$ \\
$\mathrm{X}-\mathrm{C}(1)-\mathrm{C}(6)-\mathrm{C}(5)$ & $178.74(13)$ & $178.40(19)$ \\
$\mathrm{C}(3)-\mathrm{C}(4)-\mathrm{C}(7)-\mathrm{O}$ & $-6.7(2)$ & $-6.9(4)$ \\
$\mathrm{C}(5)-\mathrm{C}(4)-\mathrm{C}(7)-\mathrm{O}$ & $171.49(16)$ & $171.3(2)$ \\
$\mathrm{C}(3)-\mathrm{C}(4)-\mathrm{C}(7)-\mathrm{C}(8)$ & $172.59(15)$ & $172.6(2)$ \\
$\mathrm{C}(5)-\mathrm{C}(4)-\mathrm{C}(7)-\mathrm{C}(8)$ & $-9.3(2)$ & $-9.3(4)$ \\
$\mathrm{O}-\mathrm{C}(7)-\mathrm{C}(8)-\mathrm{C}(9)$ & $-0.4(2)$ & $0.1(3)$ \\
$\mathrm{C}(4)-\mathrm{C}(7)-\mathrm{C}(8)-\mathrm{C}(9)$ & $-179.65(14)$ & $-179.4(2)$ \\
$\mathrm{N}(2)-\mathrm{N}(1)-\mathrm{C}(9)-\mathrm{C}(8)$ & $179.07(15)$ & $178.5(2)$ \\
$\mathrm{C}(7)-\mathrm{C}(8)-\mathrm{C}(9)-\mathrm{N}(1)$ & $178.94(14)$ & $179.2(2)$ \\
\hline $\mathrm{X}=\mathrm{Br}$ or $\mathrm{C} 1$ & &
\end{tabular}

\subsection{Table 3. N...N Intermolecular Interactions (A)}

\begin{tabular}{lll} 
& $\mathbf{1 a}$ & $\mathbf{1 b}$ \\
$\mathrm{N} 1 \ldots \mathrm{N} 1^{\mathrm{a}}$ & $3.898(1)$ & $3.952(1)$ \\
$\mathrm{N} 1 \ldots \mathrm{N} 1^{\mathrm{b}}$ & $3.757(3)$ & $3.767(5)$ \\
$\mathrm{N} 1 \ldots \mathrm{N} 3^{\mathrm{c}}$ & $3.698(3)$ & $3.694(5)$ \\
$\mathrm{N} 1 \ldots \mathrm{N} 3^{\mathrm{a}}$ & $5.278(3)$ & $5.356(4)$ \\
\hline Symmetry operations: & ${ }^{\mathrm{a}} \mathrm{x}-1, \mathrm{y}, \mathrm{z} ;{ }^{\mathrm{b}}-\mathrm{x}-1,-\mathrm{y}+1,-\mathrm{z}+2 ;{ }^{\mathrm{c}} \mathrm{x}+1, \mathrm{y}, \mathrm{z}$
\end{tabular}


1.11 Table 4. Least Squares Planes

\section{Deviation from Planarity ( $(\AA)$}

$1 \mathbf{b} 1 \mathbf{a}$

\begin{tabular}{rrr} 
C1-C2-C3-C4-C5-C6 & 0.0049 & 0.0060 \\
C7 & -0.0557 & -0.0600 \\
C8 & 0.1102 & 0.1062 \\
C9 & 0.0322 & 0.0333 \\
N1 & 0.2282 & 0.2241 \\
N2 & 0.1684 & 0.1583 \\
N3 & 0.1381 & 0.1224 \\
O & -0.2186 & -0.2300 \\
X & -0.0484 & -0.0646 \\
\hline
\end{tabular}

$\mathrm{X}=\mathrm{Br}$ or $\mathrm{Cl}$ 


\section{Molecular Modeling.}

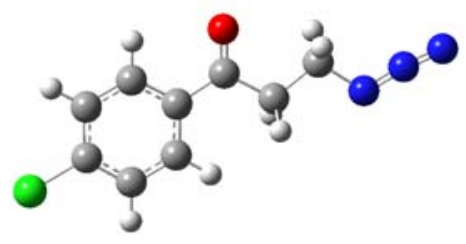

\subsection{Azide 1a}

Zero-point correction $=\quad 0.160701($ Hartree/Particle $)$

$\mathrm{E}(\mathrm{RB}+\mathrm{HF}-\mathrm{LYP})=-1047.40806826$

Standard orientation:

\begin{tabular}{|c|c|c|c|c|}
\hline \multirow{2}{*}{$\begin{array}{l}\text { Center } \\
\text { Number }\end{array}$} & \multirow{2}{*}{$\begin{array}{l}\text { Atomic } \\
\text { Number }\end{array}$} & \multirow{2}{*}{$\begin{array}{r}\text { Atomic } \\
\text { Type }\end{array}$} & \multicolumn{2}{|c|}{ Coordinates (Angstroms } \\
\hline & & & X $\quad \mathrm{Y}$ & Z \\
\hline 1 & 6 & 2.982210 & 1.120450 & 0.000000 \\
\hline 2 & 6 & 1.624373 & 1.424506 & 0.000000 \\
\hline 3 & 6 & 0.655583 & 0.406403 & 0.000000 \\
\hline 4 & 6 & 1.080211 & -0.932238 & 0.000000 \\
\hline 5 & 6 & 2.437897 & -1.252070 & 0.000000 \\
\hline 6 & 6 & 3.378217 & -0.220477 & 0.000000 \\
\hline 7 & 1 & 3.728501 & 1.908308 & 0.000000 \\
\hline 8 & 1 & 1.292553 & 2.458002 & 0.000000 \\
\hline 9 & 1 & 0.358559 & -1.743096 & 0.000000 \\
\hline 10 & 1 & 2.763858 & -2.287004 & 0.000000 \\
\hline 11 & 6 & -0.789695 & 0.799176 & 0.000000 \\
\hline 12 & 8 & -1.117383 & 1.979643 & 0.000000 \\
\hline 13 & 6 & -1.848863 & -0.295145 & 0.000000 \\
\hline 14 & 6 & -3.264547 & 0.269446 & 0.000000 \\
\hline 15 & 1 & -3.428963 & 0.895284 & 0.885132 \\
\hline 16 & 1 & -1.708968 & -0.941841 & -0.876685 \\
\hline 17 & 1 & -1.708979 & -0.941828 & 0.876696 \\
\hline 18 & 7 & -4.199140 & -0.883317 & 0.000000 \\
\hline 19 & 7 & -5.399833 & -0.595985 & 0.000000 \\
\hline 20 & 7 & -6.534193 & -0.458197 & 0.000000 \\
\hline 21 & 1 & -3.428961 & 0.895288 & -0.885132 \\
\hline 22 & 17 & 5.086548 & -0.614004 & 0.000000 \\
\hline
\end{tabular}




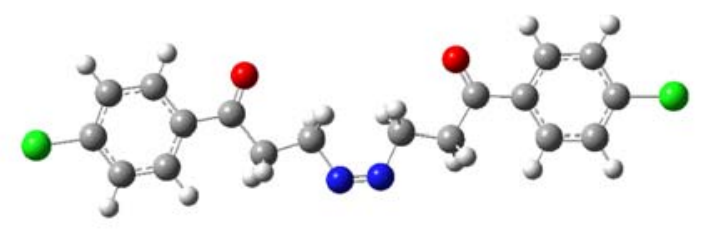

\subsection{Cis-3aA}

Zero-point correction $=0.304180$ (Hartree/Particle $)$

$\mathrm{E}(\mathrm{RB}+\mathrm{HF}-\mathrm{LYP})=-1875.88127058$

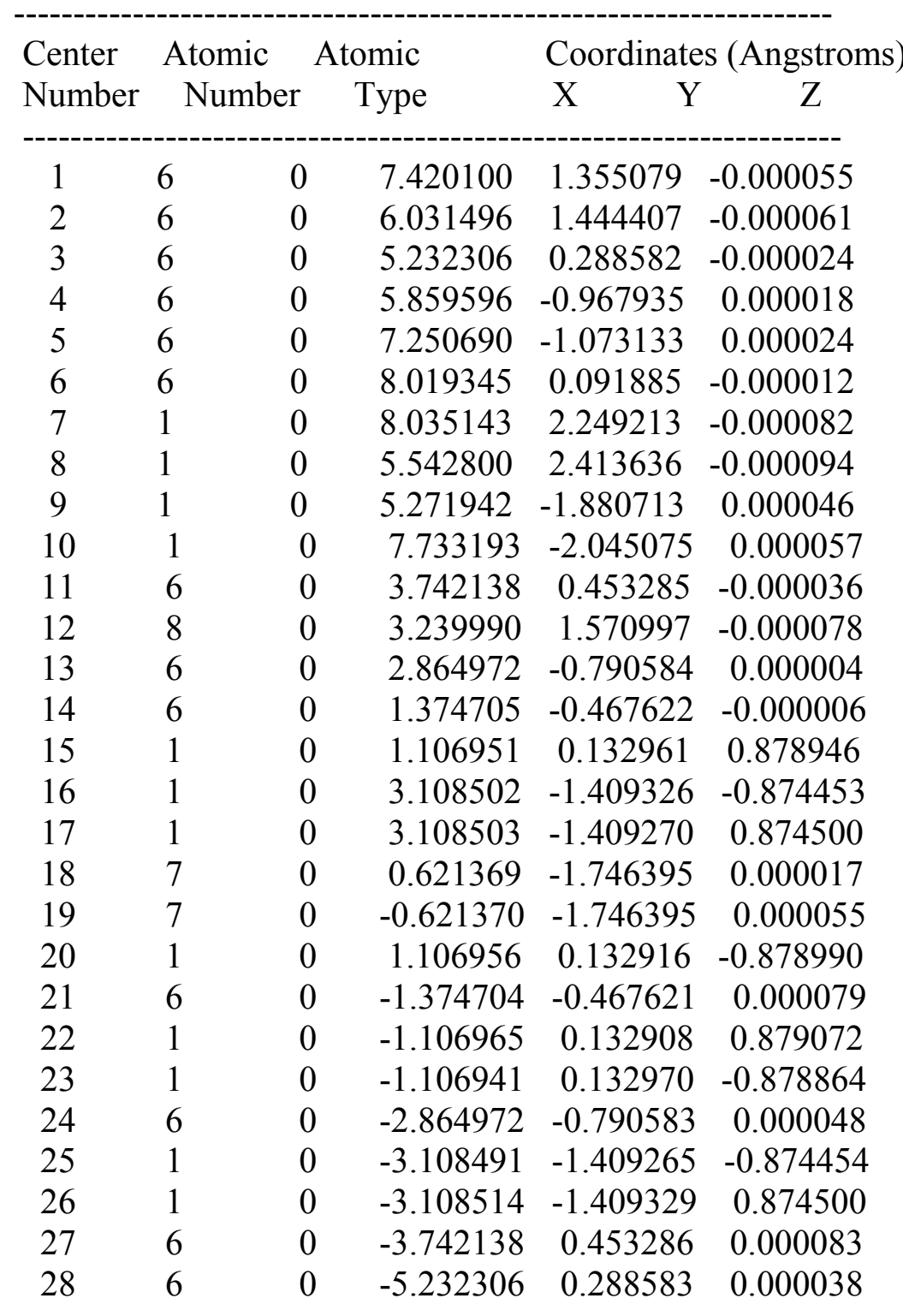




$\begin{array}{cccccc}29 & 6 & 0 & -6.031497 & 1.444407 & 0.000068 \\ 30 & 6 & 0 & -5.859595 & -0.967934 & -0.000032 \\ 31 & 6 & 0 & -7.420100 & 1.355079 & 0.000029 \\ 32 & 1 & 0 & -5.542800 & 2.413637 & 0.000122 \\ 33 & 6 & 0 & -7.250690 & -1.073133 & -0.000071 \\ 34 & 1 & 0 & -5.271941 & -1.880712 & -0.000056 \\ 35 & 6 & 0 & -8.019345 & 0.091884 & -0.000040 \\ 36 & 1 & 0 & -8.035144 & 2.249213 & 0.000052 \\ 37 & 1 & 0 & -7.733193 & -2.045075 & -0.000125 \\ 38 & 8 & 0 & -3.239990 & 1.570998 & 0.000150 \\ 39 & 17 & 0 & -9.768743 & -0.031256 & -0.000089 \\ 40 & 17 & 0 & 9.768743 & -0.031256 & -0.000005 \\ ----------------------------------------------------------------------\end{array}$

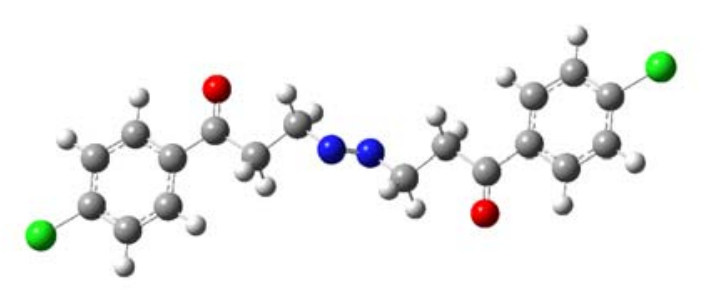

\subsection{Trans-3aA}

Zero-point correction $=0.305240$ (Hartree/Particle) $\mathrm{E}(\mathrm{RB}+\mathrm{HF}-\mathrm{LYP})=-1875.89726204$

\begin{tabular}{|c|c|c|c|c|}
\hline \multirow{2}{*}{$\begin{array}{l}\text { Center } \\
\text { Number }\end{array}$} & \multirow{2}{*}{$\begin{array}{l}\text { Atomic } \\
\text { Number }\end{array}$} & \multirow{2}{*}{$\begin{array}{r}\text { Atomic } \\
\text { Type }\end{array}$} & \multicolumn{2}{|c|}{ Coordinates (Angstrom } \\
\hline & & & $\begin{array}{ll}X & Y\end{array}$ & $\mathrm{Z}$ \\
\hline 1 & 6 & -3.666101 & -0.337322 & -6.971808 \\
\hline 2 & 6 & -3.661760 & -0.338885 & -5.580298 \\
\hline 3 & 6 & -2.454680 & -0.342371 & -4.860736 \\
\hline 4 & 6 & -1.243584 & -0.346212 & -5.571411 \\
\hline 5 & 6 & -1.232132 & -0.346835 & -6.966407 \\
\hline 6 & 6 & -2.446224 & -0.341538 & -7.654882 \\
\hline 7 & 1 & -4.599718 & -0.333335 & -7.525178 \\
\hline 8 & 1 & -4.595821 & -0.337159 & -5.027389 \\
\hline 9 & 1 & -0.293517 & -0.351313 & -5.046479 \\
\hline 10 & 1 & -0.294914 & -0.351158 & -7.513348 \\
\hline 11 & 6 & -2.519569 & -0.347070 & -3.362568 \\
\hline 12 & 8 & -3.599668 & -0.372640 & -2.787043 \\
\hline
\end{tabular}




\begin{tabular}{|c|c|c|c|c|c|}
\hline 13 & 6 & 0 & -1.216257 & -0.328079 & -2.571901 \\
\hline 14 & 6 & 0 & -1.445160 & -0.246435 & -1.061509 \\
\hline 15 & 1 & 0 & -0.598935 & 0.517095 & -2.905242 \\
\hline 16 & 1 & 0 & -0.641167 & -1.234614 & -2.808539 \\
\hline 17 & 1 & 0 & -1.890300 & 0.710751 & -0.767239 \\
\hline 18 & 1 & 0 & -2.114061 & -1.057160 & -0.751072 \\
\hline 19 & 7 & 0 & -0.162819 & -0.466330 & -0.375544 \\
\hline 20 & 7 & 0 & 0.162819 & 0.466330 & 0.375544 \\
\hline 21 & 6 & 0 & 1.445160 & 0.246435 & 1.061509 \\
\hline 22 & 6 & 0 & 1.216257 & 0.328079 & 2.571901 \\
\hline 23 & 1 & 0 & 2.114061 & 1.057160 & 0.751072 \\
\hline 24 & 1 & 0 & 1.890300 & -0.710751 & 0.767239 \\
\hline 25 & 1 & 0 & 0.641167 & 1.234614 & 2.808539 \\
\hline 26 & 1 & 0 & 0.598935 & -0.517095 & 2.905242 \\
\hline 27 & 6 & 0 & 2.519569 & 0.347070 & 3.362568 \\
\hline 28 & 8 & 0 & 3.599668 & 0.372640 & 2.787043 \\
\hline 29 & 6 & 0 & 2.454680 & 0.342371 & 4.860736 \\
\hline 30 & 6 & 0 & 3.661760 & 0.338885 & 5.580298 \\
\hline 31 & 6 & 0 & 1.243584 & 0.346212 & 5.571411 \\
\hline 32 & 6 & 0 & 3.666101 & 0.337322 & 6.971808 \\
\hline 33 & 1 & 0 & 4.595821 & 0.337159 & 5.027389 \\
\hline 34 & 6 & 0 & 1.232132 & 0.346835 & 6.966407 \\
\hline 35 & 1 & 0 & 0.293517 & 0.351313 & 5.046479 \\
\hline 36 & 6 & 0 & 2.446224 & 0.341538 & 7.654882 \\
\hline 37 & 1 & 0 & 4.599718 & 0.333335 & 7.525178 \\
\hline 38 & 1 & 0 & 0.294914 & 0.351158 & 7.513348 \\
\hline 39 & 17 & 0 & -2.441033 & -0.340177 & -9.408513 \\
\hline 40 & 17 & 0 & 2.441033 & 0.340177 & 9.408513 \\
\hline
\end{tabular}

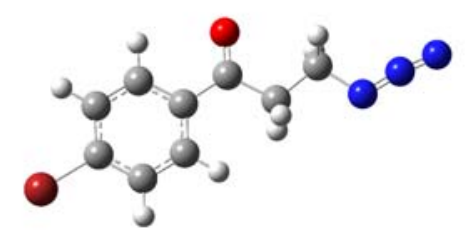

\subsection{Azide 1b}

Zero-point correction $=0.161395$ (Hartree/Particle) $\mathrm{E}(\mathrm{RB}+\mathrm{HF}-\mathrm{LYP})=-3158.94095782$

Standard orientation: 


\begin{tabular}{|c|c|c|c|c|}
\hline \multirow{2}{*}{$\begin{array}{l}\text { Center } \\
\text { Number }\end{array}$} & \multirow{2}{*}{$\begin{array}{l}\text { Atomic } \\
\text { Number }\end{array}$} & \multirow{2}{*}{$\begin{array}{l}\text { Atomic } \\
\text { Type }\end{array}$} & \multicolumn{2}{|c|}{ Coordinates (Angstroms } \\
\hline & & & X $\quad \mathrm{Y}$ & $\mathrm{Z}$ \\
\hline 1 & 6 & 2.175921 & 1.290015 & -0.000014 \\
\hline 2 & 6 & 0.809725 & 1.554961 & -0.000005 \\
\hline 3 & 6 & -0.127110 & 0.507829 & 0.000014 \\
\hline 4 & 6 & 0.336968 & -0.817347 & 0.000024 \\
\hline 5 & 6 & 1.703078 & -1.097190 & 0.000015 \\
\hline 6 & 6 & 2.611046 & -0.037947 & -0.000004 \\
\hline 7 & 1 & 2.896089 & 2.101408 & -0.000029 \\
\hline 8 & 1 & 0.447294 & 2.578187 & -0.000013 \\
\hline 9 & 1 & -0.360649 & -1.648906 & 0.000039 \\
\hline 10 & 1 & 2.056332 & -2.122744 & 0.000022 \\
\hline 11 & 6 & -1.584264 & 0.853610 & 0.000020 \\
\hline 12 & 8 & -1.950860 & 2.022530 & 0.000007 \\
\hline 13 & 6 & -2.605012 & -0.276154 & 0.000043 \\
\hline 14 & 6 & -4.039953 & 0.236603 & 0.000041 \\
\hline 15 & 1 & -4.227129 & 0.856115 & 0.885141 \\
\hline 16 & 1 & -2.442263 & -0.917624 & -0.876525 \\
\hline 17 & 1 & -2.442257 & -0.917592 & 0.876633 \\
\hline 18 & 7 & -4.931429 & -0.949733 & 0.000089 \\
\hline 19 & 7 & -6.141815 & -0.706482 & -0.000009 \\
\hline 20 & 7 & -7.280409 & -0.610070 & -0.000150 \\
\hline 21 & 1 & -4.227149 & 0.856062 & -0.885093 \\
\hline 22 & 35 & 4.477137 & -0.411070 & -0.000016 \\
\hline
\end{tabular}

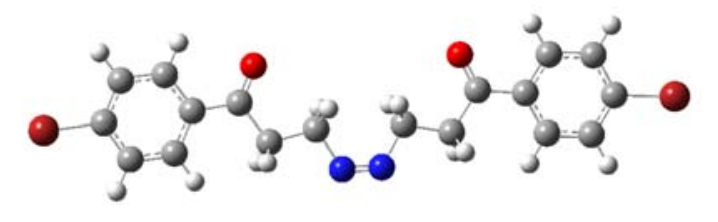

\subsection{Cis-3bA}

Zero-point correction $=\quad 0.305191($ Hartree/Particle $)$ $\mathrm{E}(\mathrm{RB}+\mathrm{HF}-\mathrm{LYP})=-6098.94692184$

Standard orientation:

Center Atomic Atomic Coordinates (Angstroms) 


\begin{tabular}{|c|c|c|c|c|c|}
\hline Number & & & Type & $\mathrm{X}$ & Z \\
\hline 1 & 6 & 0 & -7.422882 & 1.360981 & -0.000014 \\
\hline 2 & 6 & 0 & -6.034772 & 1.458319 & 0.000064 \\
\hline 3 & 6 & 0 & -5.231785 & 0.305520 & 0.000090 \\
\hline 4 & 6 & 0 & -5.852593 & -0.953778 & 0.000038 \\
\hline 5 & 6 & 0 & -7.242904 & -1.065924 & -0.000042 \\
\hline 6 & 6 & 0 & -8.015663 & 0.095464 & -0.000069 \\
\hline 7 & 1 & 0 & -8.039794 & 2.253556 & -0.000034 \\
\hline 8 & 1 & 0 & -5.550475 & 2.429783 & 0.000105 \\
\hline 9 & 1 & 0 & -5.260362 & -1.863576 & 0.000057 \\
\hline 10 & 1 & 0 & -7.717591 & -2.041353 & -0.000082 \\
\hline 11 & 6 & 0 & -3.742211 & 0.473855 & 0.000166 \\
\hline 12 & 8 & 0 & -3.242124 & 1.592459 & 0.000179 \\
\hline 13 & 6 & 0 & -2.864087 & -0.768850 & 0.000104 \\
\hline 14 & 6 & 0 & -1.374187 & -0.445833 & 0.000114 \\
\hline 15 & 1 & 0 & -1.106289 & 0.154711 & -0.878850 \\
\hline 16 & 1 & 0 & -3.107239 & -1.387858 & 0.874461 \\
\hline 17 & 1 & 0 & -3.107248 & -1.387772 & -0.874310 \\
\hline 18 & 7 & 0 & -0.621338 & -1.724689 & 0.000072 \\
\hline 19 & 7 & 0 & 0.621338 & -1.724689 & 0.000081 \\
\hline 20 & 1 & 0 & -1.106289 & 0.154648 & 0.879121 \\
\hline 21 & 6 & 0 & 1.374187 & -0.445833 & 0.000146 \\
\hline 22 & 1 & 0 & 1.106340 & 0.154689 & -0.878849 \\
\hline 23 & 1 & 0 & 1.106238 & 0.154670 & 0.879122 \\
\hline 24 & 6 & 0 & 2.864087 & -0.768850 & 0.000220 \\
\hline 25 & 1 & 0 & 3.107195 & -1.387827 & 0.874612 \\
\hline 26 & 1 & 0 & 3.107292 & -1.387803 & -0.874160 \\
\hline 27 & 6 & 0 & 3.742211 & 0.473855 & 0.000257 \\
\hline 28 & 6 & 0 & 5.231785 & 0.305520 & 0.000150 \\
\hline 29 & 6 & 0 & 6.034772 & 1.458319 & 0.000045 \\
\hline 30 & 6 & 0 & 5.852593 & -0.953778 & 0.000163 \\
\hline 31 & 6 & 0 & 7.422882 & 1.360981 & -0.000065 \\
\hline 32 & 1 & 0 & 5.550475 & 2.429783 & 0.000043 \\
\hline 33 & 6 & 0 & 7.242904 & -1.065924 & 0.000056 \\
\hline 34 & 1 & 0 & 5.260362 & -1.863576 & 0.000254 \\
\hline 35 & 6 & 0 & 8.015663 & 0.095464 & -0.000056 \\
\hline 36 & 1 & 0 & 8.039794 & 2.253556 & -0.000154 \\
\hline 37 & 1 & 0 & 7.717591 & -2.041353 & 0.000061 \\
\hline 38 & 8 & 0 & 3.242124 & 1.592459 & 0.000155 \\
\hline 39 & 35 & 0 & 9.913636 & -0.049643 & -0.000210 \\
\hline 40 & 35 & 0 & -9.913636 & -0.049643 & -0.000171 \\
\hline
\end{tabular}




\subsection{Cis-3bB}

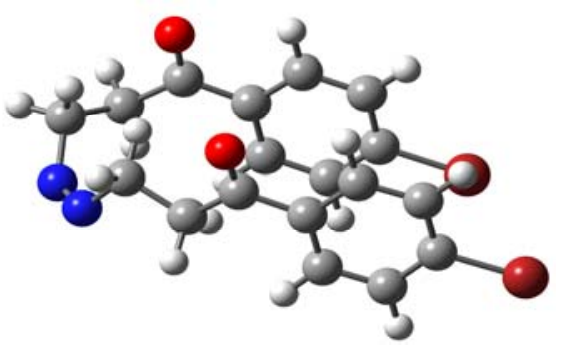

Zero-point correction $=0.305632($ Hartree/Particle $)$ $\mathrm{E}(\mathrm{RB}+\mathrm{HF}-\mathrm{LYP})=-6098.941715565$

Standard orientation:

\begin{tabular}{|c|c|c|c|c|}
\hline \multirow{2}{*}{$\begin{array}{l}\text { Center } \\
\text { Number }\end{array}$} & Atomic & \multicolumn{3}{|c|}{ Forces (Hartrees/Bohr) } \\
\hline & & Jumber & $\mathrm{Y}$ & $\mathrm{Z}$ \\
\hline 1 & 6 & -0.000000082 & -0.000000799 & 0.000000893 \\
\hline 2 & 6 & -0.000002084 & -0.000000160 & -0.000001874 \\
\hline 3 & 6 & -0.000002010 & -0.000004110 & 0.000001430 \\
\hline 4 & 6 & -0.000000311 & 0.000001377 & -0.000000987 \\
\hline 5 & 6 & -0.000000564 & -0.000001115 & -0.000001116 \\
\hline 6 & 6 & -0.000002444 & 0.000002880 & 0.000000396 \\
\hline 7 & 1 & -0.000000140 & 0.000001903 & -0.000002511 \\
\hline 8 & 1 & -0.000002408 & 0.000001965 & -0.000002790 \\
\hline 9 & 1 & -0.000002620 & -0.000001461 & -0.000001455 \\
\hline 10 & 1 & 0.000000042 & -0.000001679 & 0.000001944 \\
\hline 11 & 6 & -0.000001458 & 0.000010190 & -0.000003324 \\
\hline 12 & 8 & -0.000003347 & -0.000000150 & -0.000000670 \\
\hline 13 & 6 & -0.000002861 & -0.000001832 & -0.000001180 \\
\hline 14 & 6 & -0.000004161 & 0.000004530 & -0.000000361 \\
\hline 15 & 1 & -0.000007080 & -0.000006371 & 0.000005832 \\
\hline 16 & 1 & -0.000004484 & -0.000001528 & 0.000001589 \\
\hline 17 & 1 & -0.000002463 & -0.000007103 & 0.000000397 \\
\hline 18 & 1 & -0.000003823 & -0.000001799 & -0.000000307 \\
\hline 19 & 7 & -0.000003768 & -0.000007719 & 0.000007359 \\
\hline 20 & 7 & -0.000001110 & 0.000003111 & 0.000000211 \\
\hline 21 & 6 & -0.000008708 & -0.000005219 & -0.000002686 \\
\hline 22 & 6 & 0.000002927 & 0.000000326 & 0.000006081 \\
\hline 23 & 1 & 0.000001028 & 0.000000721 & -0.000001420 \\
\hline 24 & 1 & -0.000001047 & 0.000002958 & -0.000001529 \\
\hline 25 & 1 & 0.000002372 & -0.000001794 & -0.000001269 \\
\hline 26 & 1 & 0.000001992 & 0.000000212 & -0.000001076 \\
\hline 27 & 6 & -0.000002246 & 0.000000338 & 0.000006163 \\
\hline 28 & 8 & 0.000002464 & 0.000001115 & -0.000002524 \\
\hline 29 & 6 & 0.000004567 & 0.000000555 & -0.000002433 \\
\hline 30 & 6 & 0.000003192 & 0.000001732 & -0.000000131 \\
\hline
\end{tabular}




$\begin{array}{rcrrr}31 & 6 & 0.000003867 & -0.000001531 & 0.000000332 \\ 32 & 6 & 0.000006295 & -0.000000145 & -0.000003095 \\ 33 & 1 & 0.000002955 & 0.000002875 & -0.000001860 \\ 34 & 6 & 0.000003466 & -0.000001130 & 0.000001238 \\ 35 & 1 & 0.000002980 & -0.000000652 & 0.000002247 \\ 36 & 6 & 0.000002310 & 0.000004590 & -0.000000294 \\ 37 & 1 & 0.000004650 & 0.000003241 & -0.000002044 \\ 38 & 1 & 0.000004742 & 0.000000087 & 0.000001567 \\ 39 & 35 & 0.000006599 & 0.000001881 & -0.000001418 \\ 40 & 35 & 0.000002771 & -0.000000292 & 0.000000675\end{array}$

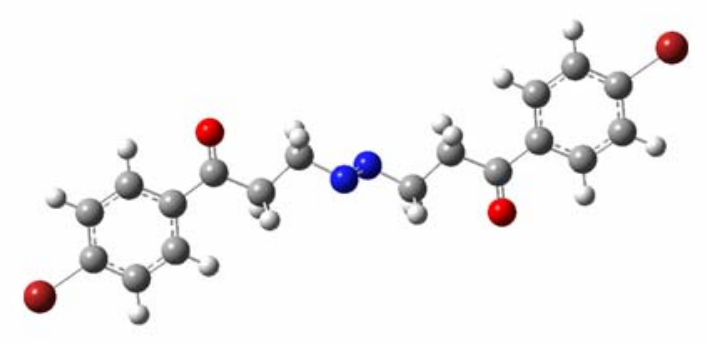

\subsection{Trans-3bA}

$\mathrm{E}(\mathrm{RB}+\mathrm{HF}-\mathrm{LYP})=-6098.96284687$

Zero-point correction $=\quad 0.306029($ Hartree/Particle $)$

Standard orientation:

\begin{tabular}{|c|c|c|c|c|}
\hline \multirow{2}{*}{$\begin{array}{l}\text { Center } \\
\text { Number }\end{array}$} & \multirow{2}{*}{$\begin{array}{l}\text { Atomic } \\
\text { Number }\end{array}$} & \multirow{2}{*}{$\begin{array}{l}\text { Atomic } \\
\text { Type }\end{array}$} & \multicolumn{2}{|c|}{ Coordinates (Angstroms } \\
\hline & & & X $\quad \mathrm{Y}$ & $\mathrm{Z}$ \\
\hline 1 & 6 & -3.564797 & -0.156351 & -7.030920 \\
\hline 2 & 6 & -3.586501 & -0.170319 & -5.639497 \\
\hline 3 & 6 & -2.392486 & -0.215477 & -4.900537 \\
\hline 4 & 6 & -1.169722 & -0.247575 & -5.589524 \\
\hline 5 & 6 & -1.132898 & -0.235246 & -6.983647 \\
\hline 6 & 6 & -2.333932 & -0.188986 & -7.691751 \\
\hline 7 & 1 & -4.489239 & -0.120744 & -7.598010 \\
\hline 8 & 1 & -4.529756 & -0.146492 & -5.102867 \\
\hline 9 & 1 & -0.229811 & -0.285220 & -5.047865 \\
\hline 10 & 1 & -0.185218 & -0.261612 & -7.511078 \\
\hline 11 & 6 & -2.480382 & -0.231388 & -3.403628 \\
\hline 12 & 8 & -3.569092 & -0.229621 & -2.844005 \\
\hline 13 & 6 & -1.188544 & -0.258649 & -2.595293 \\
\hline 14 & 6 & -1.434963 & -0.182830 & -1.087574 \\
\hline 15 & 1 & -0.540793 & 0.569546 & -2.913012 \\
\hline 16 & 1 & -0.638337 & -1.180582 & -2.831738 \\
\hline
\end{tabular}




$\begin{array}{cccccc}17 & 1 & 0 & -1.850533 & 0.786409 & -0.789488 \\ 18 & 1 & 0 & -2.136250 & -0.972366 & -0.794203 \\ 19 & 7 & 0 & -0.171042 & -0.454603 & -0.386002 \\ 20 & 7 & 0 & 0.171042 & 0.454603 & 0.386002 \\ 21 & 6 & 0 & 1.434963 & 0.182830 & 1.087574 \\ 22 & 6 & 0 & 1.188544 & 0.258649 & 2.595293 \\ 23 & 1 & 0 & 2.136250 & 0.972366 & 0.794203 \\ 24 & 1 & 0 & 1.850533 & -0.786409 & 0.789488 \\ 25 & 1 & 0 & 0.638337 & 1.180582 & 2.831738 \\ 26 & 1 & 0 & 0.540793 & -0.569546 & 2.913012 \\ 27 & 6 & 0 & 2.480382 & 0.231388 & 3.403628 \\ 28 & 8 & 0 & 3.569092 & 0.229621 & 2.844005 \\ 29 & 6 & 0 & 2.392486 & 0.215477 & 4.900537 \\ 30 & 6 & 0 & 3.586501 & 0.170319 & 5.639497 \\ 31 & 6 & 0 & 1.169722 & 0.247575 & 5.589524 \\ 32 & 6 & 0 & 3.564797 & 0.156351 & 7.030920 \\ 33 & 1 & 0 & 4.529756 & 0.146492 & 5.102867 \\ 34 & 6 & 0 & 1.132898 & 0.235246 & 6.983647 \\ 35 & 1 & 0 & 0.229811 & 0.285220 & 5.047865 \\ 36 & 6 & 0 & 2.333932 & 0.188986 & 7.691751 \\ 37 & 1 & 0 & 4.489239 & 0.120744 & 7.598010 \\ 38 & 1 & 0 & 0.185218 & 0.261612 & 7.511078 \\ 39 & 35 & 0 & 2.291754 & 0.171755 & 9.594389 \\ 40 & 35 & 0 & -2.291754 & -0.171755 & -9.594389 \\ ---------------------------------------------------------\end{array}$

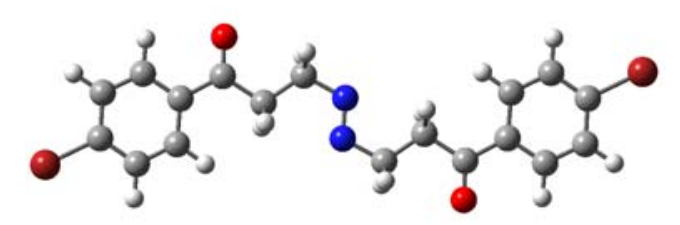

\subsection{Trans-3bB}

$\mathrm{E}(\mathrm{RB}+\mathrm{HF}-\mathrm{LYP})=-6098.96004268$

Zero-point correction $=\quad 0.306063($ Hartree/Particle $)$

Standard orientation:

\begin{tabular}{lcccc} 
Center & \multicolumn{2}{c}{ Atomic } & \multicolumn{3}{c}{ Forces $($ Hartrees/Bohr) } \\
Number & Number & X & Y & Z \\
-1 & 6 & -0.000601962 & -0.000351353 & -0.000015343
\end{tabular}




$\begin{array}{ccccc}2 & 6 & 0.000400421 & 0.000090212 & -0.000020758 \\ 3 & 6 & -0.000422156 & -0.000281501 & 0.000023006 \\ 4 & 6 & -0.000071457 & 0.000140028 & 0.000039810 \\ 5 & 6 & -0.000093968 & 0.000087772 & 0.000013578 \\ 6 & 6 & 0.000756757 & 0.000470993 & 0.000001129 \\ 7 & 1 & 0.000136564 & 0.000063553 & 0.000000882 \\ 8 & 1 & -0.000040558 & 0.000012856 & 0.000014169 \\ 9 & 1 & 0.000108858 & -0.000006594 & -0.000036172 \\ 10 & 1 & -0.000016574 & -0.000022445 & -0.000006755 \\ 11 & 6 & 0.000555162 & 0.002149628 & -0.000016388 \\ 12 & 8 & -0.000249268 & -0.002010743 & -0.000005848 \\ 13 & 6 & 0.000182115 & -0.000347903 & 0.000015446 \\ 14 & 6 & 0.000140982 & 0.000171423 & -0.000006057 \\ 15 & 1 & -0.000045525 & 0.000051776 & -0.000127205 \\ 16 & 1 & -0.000010549 & 0.000048315 & 0.000108372 \\ 17 & 1 & 0.000079962 & 0.000090350 & 0.000146634 \\ 18 & 1 & 0.000083007 & 0.000089929 & -0.000132322 \\ 19 & 7 & -0.000382355 & -0.000196066 & -0.000035901 \\ 20 & 7 & -0.000411794 & -0.000202471 & 0.000034107 \\ 21 & 6 & 0.000045893 & 0.000213264 & 0.000009570 \\ 22 & 6 & 0.000333349 & -0.000293738 & -0.000016460 \\ 23 & 1 & 0.000077588 & 0.000064115 & -0.000172781 \\ 24 & 1 & 0.000081453 & 0.000063600 & 0.000157572 \\ 25 & 1 & -0.000028046 & 0.000020637 & 0.000120220 \\ 26 & 1 & 0.000006222 & 0.000017739 & -0.000100740 \\ 27 & 6 & 0.000486964 & 0.002166529 & 0.000015541 \\ 28 & 8 & -0.000244879 & -0.002069540 & 0.000006391 \\ 29 & 6 & -0.000543770 & -0.000330632 & -0.000022967 \\ 30 & 6 & 0.000334189 & 0.000075743 & 0.000020638 \\ 31 & 6 & -0.000053469 & 0.000104686 & -0.000041214 \\ 32 & 6 & -0.000585760 & -0.000350515 & 0.000014530 \\ 33 & 1 & -0.000064798 & 0.000014138 & -0.000013530 \\ 34 & 6 & -0.000006735 & 0.000077737 & -0.000014593 \\ 35 & 1 & 0.000141440 & 0.000120854 & 0.000037202 \\ 36 & 6 & 0.000816895 & 0.000534015 & -0.000001201 \\ 37 & 1 & 0.000080729 & 0.000132908 & -0.000000183 \\ 38 & 1 & -0.000089532 & -0.000080227 & 0.000007743 \\ 39 & 35 & -0.000415922 & -0.000262612 & 0.000002045 \\ 40 & 35 & -0.000469472 & -0.000266461 & -0.000002165\end{array}$




\section{NMR, IR and MS Spectra for 4a,b}

1-(4-bromophenyl)-3-[5-(4-bromo-phenyl)pyrazol-1-yl]-propan-1-one

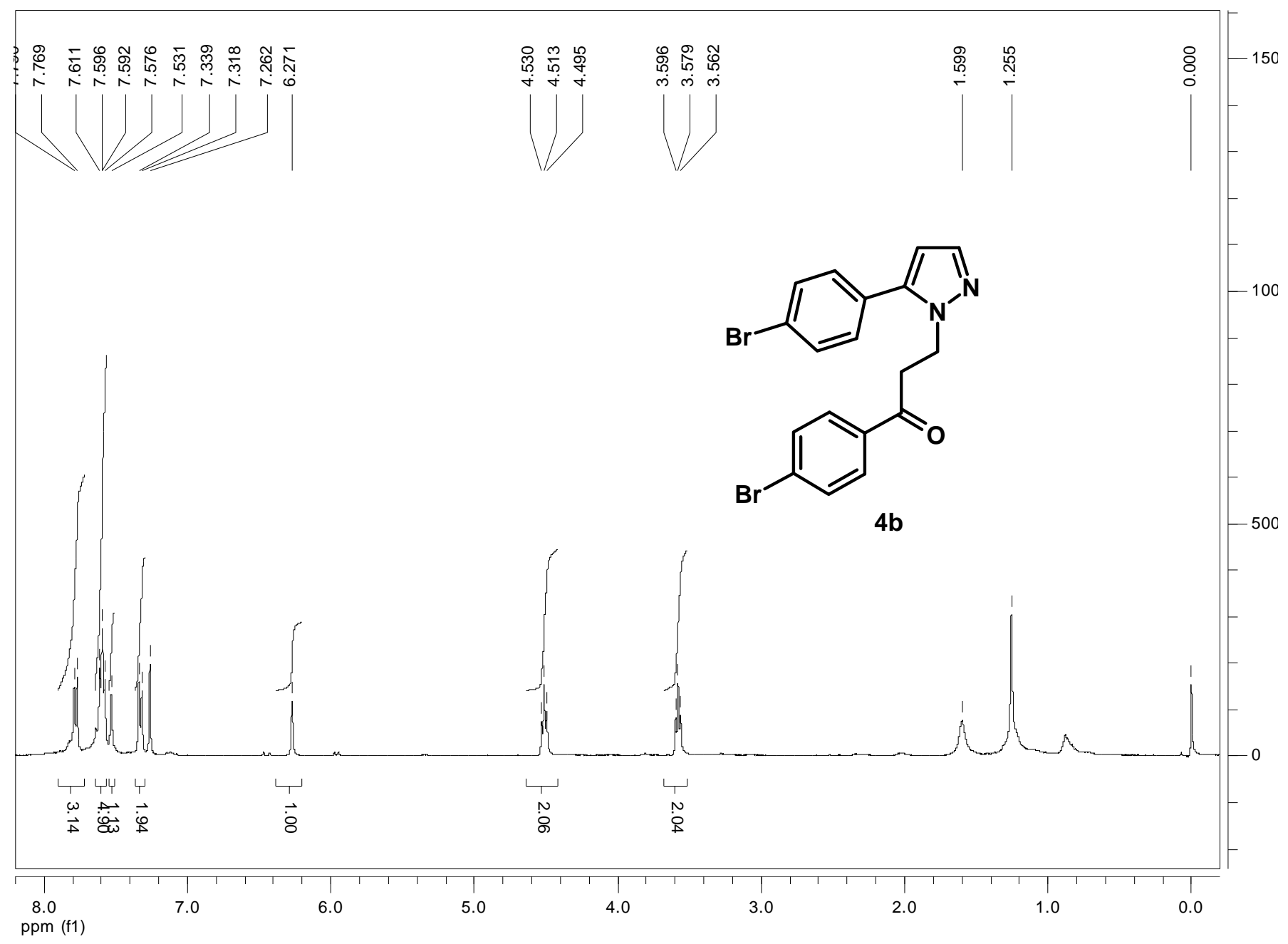

Figure S5. ${ }^{1} \mathrm{H}-\mathrm{NMR}$ spectrum of $\mathbf{4 b}$. 


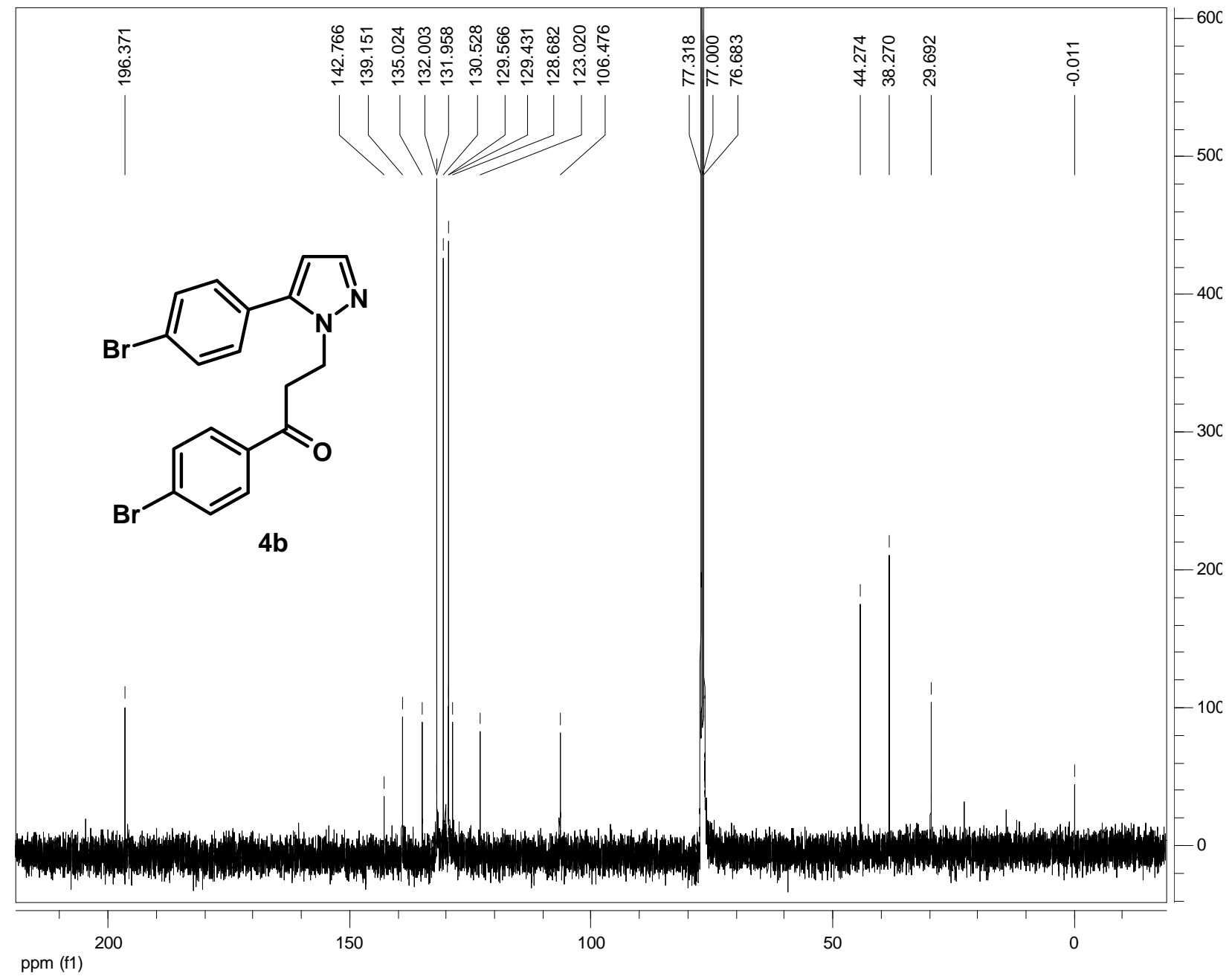

Figure S6. ${ }^{13} \mathrm{C}$-NMR spectrum of $\mathbf{4 b}$. 


\section{IonSpec HiResES|}

File: kokajms20030909_0002_ESl.trans

003522. pbrf3a. SM/ADG/Chem.

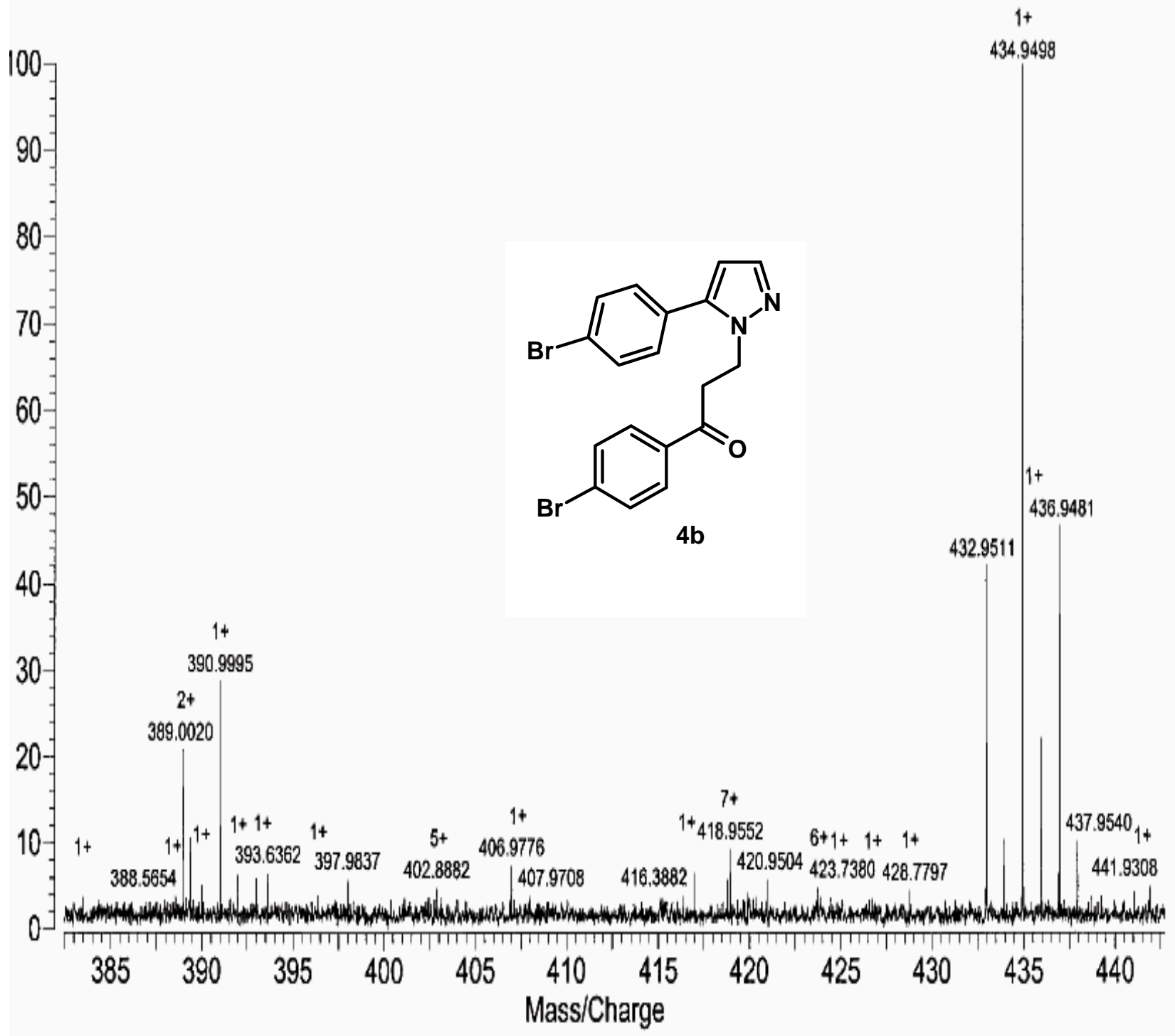

Figure S7. High Resolution MS spectrum for $\mathbf{4 b}$. 


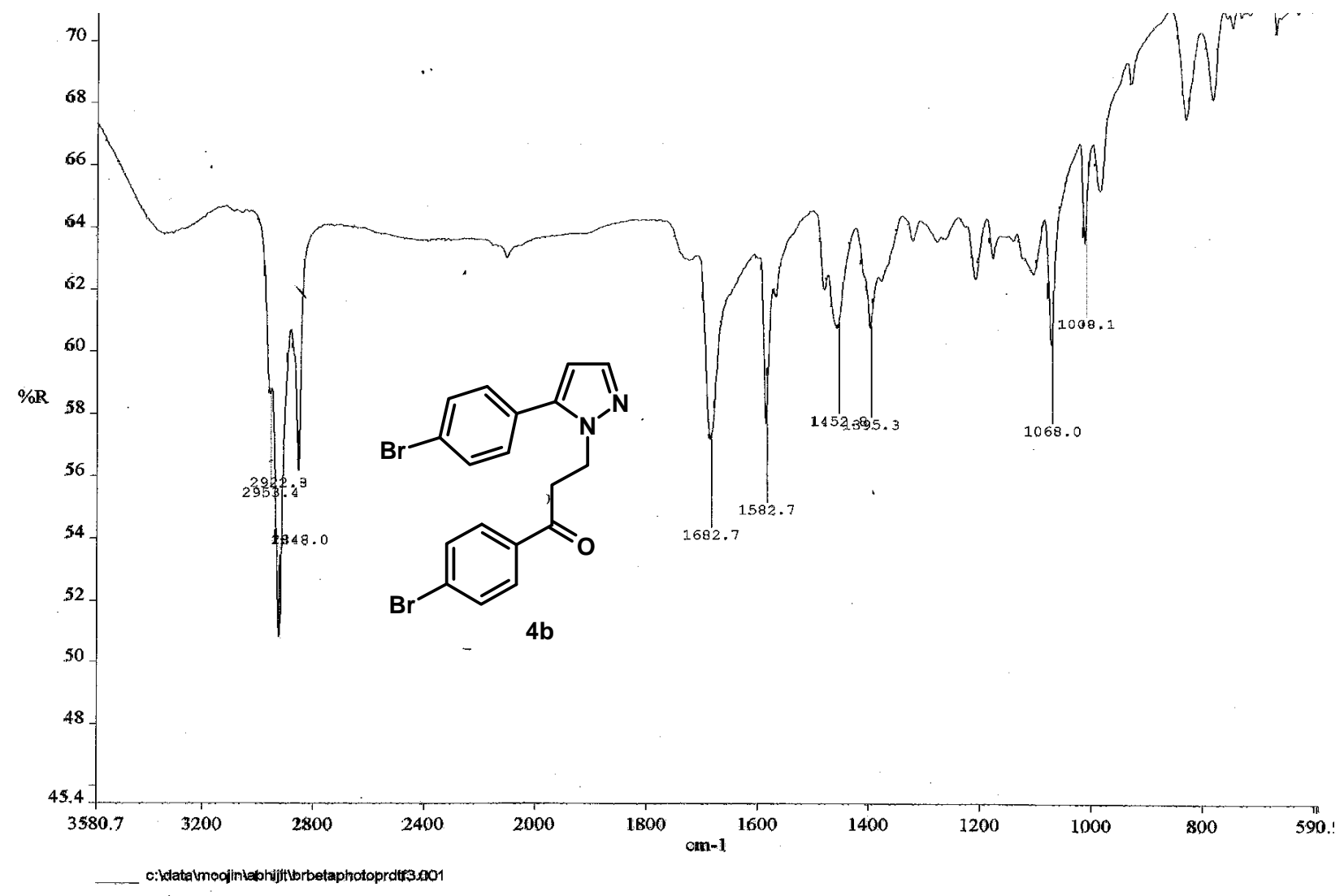

Figure S8. IR spectrum of $\mathbf{4 b}$. 


\section{1-(4-chlorophenyl)-3-[5-(4-chloro-phenyl)pyrazol-1-yl]-propan-1-one}

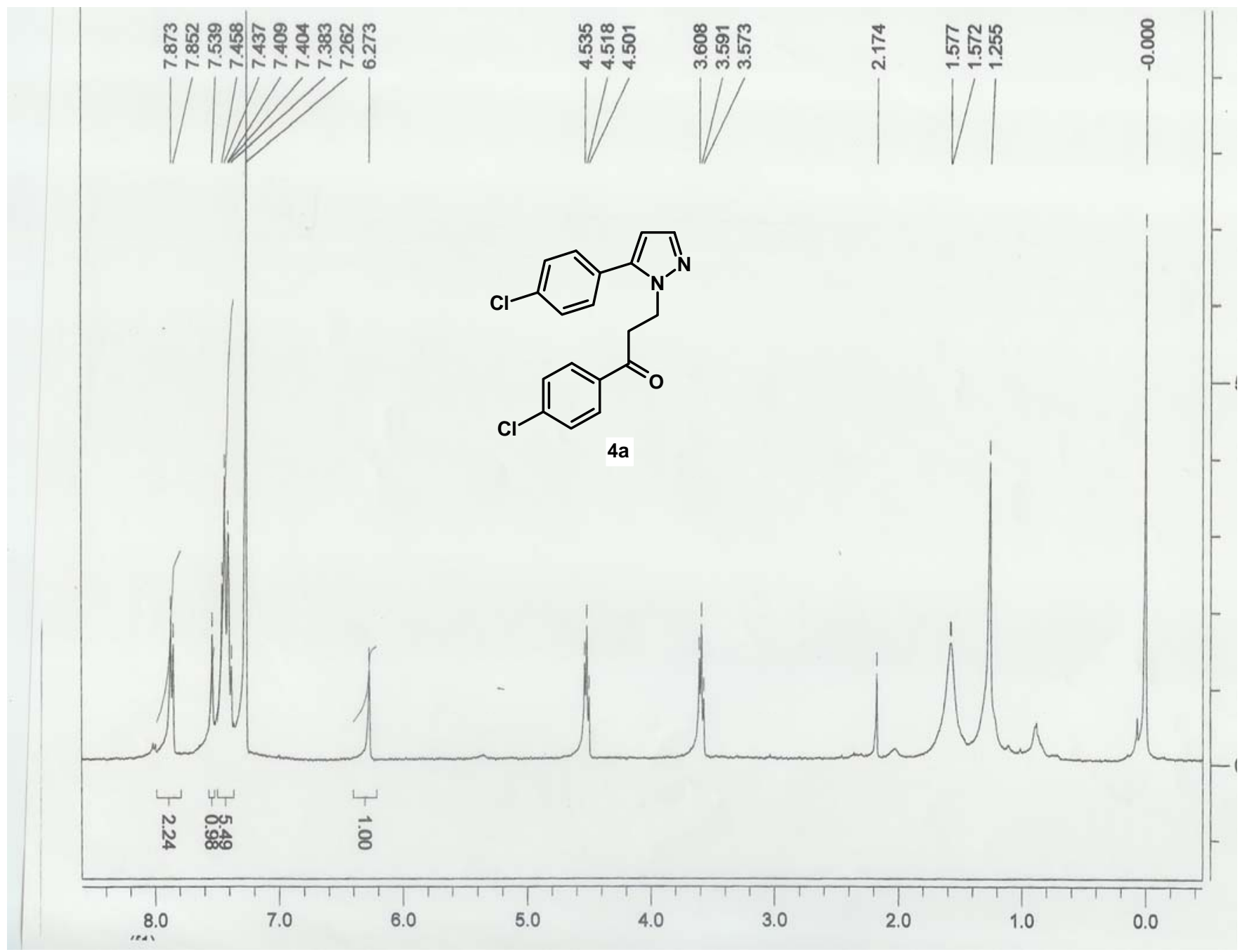

Figure S9. ${ }^{1} \mathrm{H}-\mathrm{NMR}$ spectrum of $\mathbf{4 a}$. 


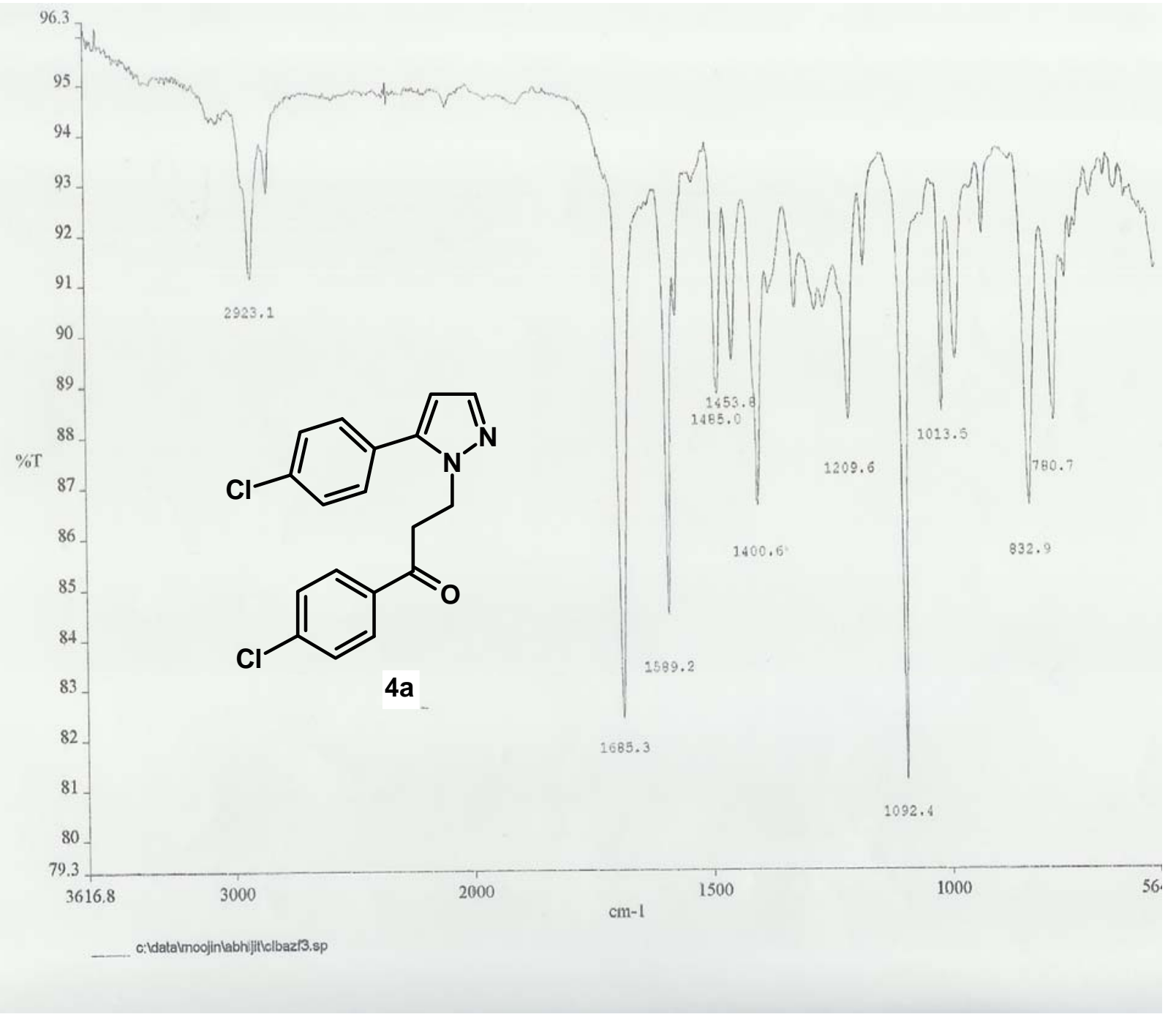

Figure S10. IR spectrum of $\mathbf{4 a}$. 